\title{
A 3D Multi-frequency response electrical mesh phantom for validation of the planar structure EIT system performance
}

\author{
A. Zarafshani ${ }^{1}$, T. Qureshi ${ }^{1}$, T. Bach ${ }^{2}$, C.R. Chatwin ${ }^{1}$ and M. Soleimani ${ }^{3}$ \\ ${ }^{1}$ Instrumentation and Hardware, Bioelectrical Research Group, Engineering and Design, University of Sussex, England, \\ BN1 9QT \\ ${ }^{2}$ Sensatech Research, Brighton, England, BN2 0GP \\ ${ }^{3}$ Engineering Tomography Laboratory, Electronics and Electrical Engineering, University of Bath, England, BA2 7AY
} a.zarafshani@sussex.ac.uk

\begin{abstract}
Assessment and validation of the Electrical Impedance Tomography (EIT) system performance and calibration of systematic errors in the electrical field generated inside of the interrogated volume is an important requirement. System instabilities can be caused by the EIT design and must be characterized before and during the clinical trials. Evaluation of the Sussex EIT system used in the clinical study can be based on a realistic electronic phantom. We designed a mesh phantom based on the electrode configuration and mesh structures of the image reconstruction. The phantom has the capability of modelling the cellular electrical properties that are operative within a circular homogeneous medium. The design is optimized to assess the planar topology of the internal impedance distribution. The system employs the information from the electrical properties of biological tissues to evaluate the Cole-Cole dispersion data. This mesh phantom is capable of producing localized conductivity perturbations between each arbitrary channel in the electrode placement planar phantom topology by measuring all $\mathbf{1 4 1 6}$ combinations that are to be used in the image reconstruction. The phantom is especially designed for the Sussex EIT system to validate system performance of measurements consisting of SNR, and modelling system accuracy.
\end{abstract}

\section{INTRODUCTION}

Essentially, the permittivity is a measure of the ability to permit the storage of electrical energy in the medium. Electrical Impedance Tomography (EIT), in biomedical engineering, is a medical imaging technique that maps the conductivity or permittivity distribution inside a biological tissue sample, by injecting current or applying a voltage to the tissue and collecting voltages or currents at the subject surface $[1,2]$. EIT systems use impedance data for imaging biological tissues using the change of impedance brought about by disease states [2].

The EIT technique is one of the most effective non-invasive modalities that could be used for detection or screening breast cancer. EIT systems are able to distinguish cancerous abnormal tissue from normal tissue using their different impedance properties and to find the impedance distribution of the target [3].

The Sussex EIT system connects an array of 85 electrodes to the target using a medium having its own and homogeneous conductivity distribution. It then chooses two electrodes and injects in-phase and 180 degree out-of-phase constant currents and chooses two other electrodes to measure the differential voltage between them [4-7]. It does this for different injecting and receiving electrode pairs collecting the data into an array and then uses a numerical inverse algorithm to find the impedance distribution that could have given such data [6-8].

In general the mesh phantoms are designed to assess and evaluate the performance of the EIT system by providing reconfigurable conductivity distributions [9]. Using a mesh phantom can reduce the difficulty of calibration and validation of the design as it is relatively simple and quick to use $[10,11]$.

There are two phantom types to assess an EIT system: physical and electronic phantoms. The physical phantom that is used for EIT systems typically is composed of a conductive gel medium with objects embedded in this medium [12-14]. The medium and object have different impedances, which permit the EIT system to detect changes of impedance and image them [12, 15]. Physical phantoms composed of a conductive medium of saline solution with insulator, agar or vegetable, have common problematic issues of: short life, inflexibility, instability and uncontrollable physical characteristics $[11,15,16]$. The electronic phantom (Ephantom) is used to assess the system characteristics of the EIT system such as: current or voltage delivered, internal stray impedance and effects of the impedance of the medium on the measurements $[17,18]$. These results can then be used to optimise the electronic circuitry and components relevant to the impedance measurements to improve the performance of the system [19, 20]. The advantages of an E-phantom compared with the physical phantom are: improved quality, reproducibility, predictability and stability of signals $[10,12$, $15,21,22]$. 


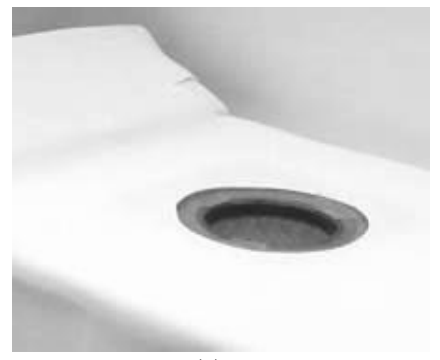

(a)

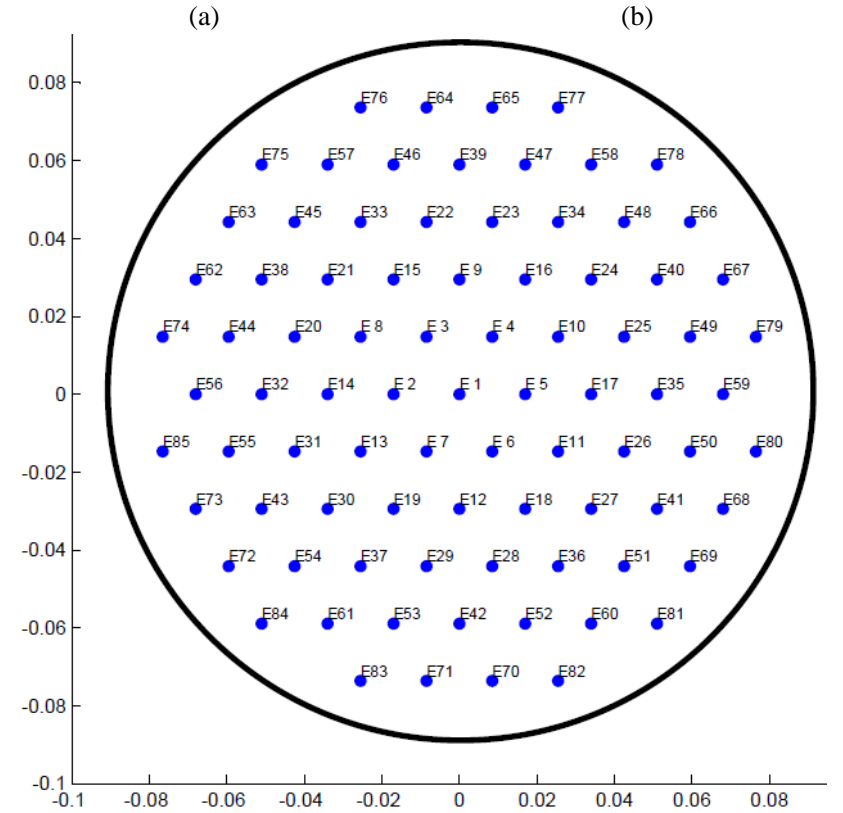

(c)

Fig. 1(a) Measurement tank $180 \mathrm{~mm}$ diameter inside the clinical bed, open top surface, $5 \mathrm{~mm}$ thick acrylic walls, (b) shows a 3D mesh of the saline tank with tetrahedral elements. The 3D tank and electrode structures are constructed in FreeCAD software to generate a homogenous mesh phantom and (c) a planar 85-electrodes structure located at the bottom of the tank.

\section{PHANTOM CONCEPTS}

The concept of the phantom here is to use a non-biological device to simulate a biological medium and giving similar biological behaviour. This E-phantom is capable of simulating biological items such as pieces of carcinoma, fat, and stroma tissues in a saline based solution. A saline conductivity of $0.5 \mathrm{mS} / \mathrm{cm}$ has been used as the homogeneous medium in order to give a low impedance connection from sensor electrodes to the breast target as in viva condition. This study uses an electrical mesh phantom, in contrast to the use of a physical phantom, to mimic an electrical impedance distribution of biological objects for comparison of different methods to improve the numerical inversion and EIT hardware and for validation of the inverse impedance image evaluated.

The Sussex EIM (Electrical Impedance Mammography) system is especially designed for breast cancer detection application for different breast sizes and shapes [6]. It is fitted in a bed with the patient assessed whilst in a prone position, as shown in Fig. 1.

\section{Phantom Methodology}

In order to design a mesh phantom for a planar electrode array with a homogeneous conductivity distribution (i.e. a conductive homogenous saline solution or permittivity properties), a Finite Element Method (FEM) over the electrode array has been applied to solve for the electrical conductivity field by registering all FEM element matrixes with the electrode array. It uses the discretization domain process over the entire domain of the continuous conductive medium $\Omega$. It works by dividing the domain into small sub-domains called elements.

It then solves the differential equations for each tetrahedral element and gathering all elements with the compatibility and equilibrium conditions for the entire domain. The geometry of electrodes and nodes present in the FEM is preserved in the physical phantom made up of resistors. Therefore, in order to make it easy to calculate the value of the resistors, the FEM is used to calculate the conductivity of each element in the matrix; then these conductivity elements are replaced by resistors with the same conductance. We have employed a 3D mesh of the homogenous conductivity medium that was generated in FreeCAD software and used in the image reconstruction program. Thus the Laplace equation is applied as follows:

$$
\begin{gathered}
\nabla . \sigma . \nabla U=0 \\
(U)=\frac{1}{2} \int_{\Omega} \sigma \nabla U . \nabla U d \Omega
\end{gathered}
$$

where $\nabla \mathrm{U}=\mathrm{U}_{\mathrm{i}} \cdot \nabla \alpha_{\mathrm{i}}$ thus,

$$
F(U)=\frac{1}{2} \sigma U_{i} \int \nabla \alpha_{i} \nabla \alpha_{j} d \Omega U_{j}=\frac{1}{2} U^{T} Y_{i j} U
$$

The global matrix [Y] is assembled from all element admittances $\left[\mathrm{Y}^{\mathrm{e}}\right]$. Thus, we can evaluate the conductivity distribution and build a global matrix of resistors for the phantom.

\section{PHANTOM BOARD}

An E-phantom PCB was built based on the FEM design that has been explained in the previous section; the mesh phantom was generated in FreeCAD software that is exploited in the reconstruction algorithm. A MATLAB program based on the same framework of elements is used. The MATLAB program produced a global admittance matrix with each element of this admittance matrix assigned to each edge of the mesh. Fig. 2(a) shows the E-phantom PCB designed. The PCB is fabricated on a circular board with an $83 \mathrm{~mm}$ radius fitted with resistors of $0.1 \%$ tolerance and temperature coefficient of $\pm 25 \mathrm{ppm} /{ }^{\circ} \mathrm{C}$. In some cases each two resistors are matched to an equivalent resistor $(\mathrm{REQ}=\mathrm{RX} \| \mathrm{RY})$ with a tolerance target of $\pm 0.2 \%$. The E-phantom connects to the EIT electrodes by means of 85 spring-loaded, stainless steel electrodes with gold plated tips. 


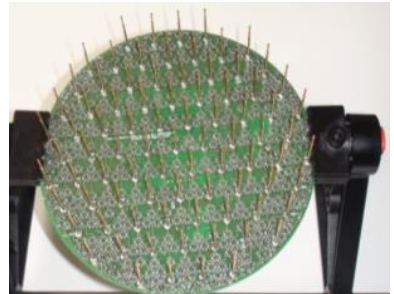

(a)

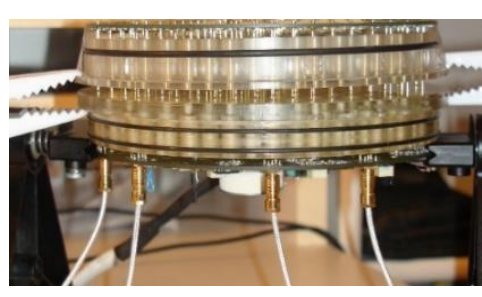

(b)
Fig. 2(a) The Mesh Phantom PCB, (b) shows the direct connection between the EIT board at the bottom and the E-phantom at the top through 85 electrodes with internal resistance less than $20 \mathrm{~m} \Omega$.

The electrodes are exactly the same as the electrodes used in the real EIT system. However, we have not taken into consideration the size, shape and material of the electrodes in the FEM used to specify the resistors required for the Ephantom. We don't need to reproduce the complex impedance behaviour of the electrodes or using an equivalent electrical model (a parallel combination of a resistor and a capacitor) of electrodes because we have employed the actual electrodes directly connected to the EIT board and E-phantom as shown in Fig. 2(b), thus we have not ignored the complex impedance behaviour of the electrodes as we directly connect the EIT electrode plate to the E-phantom board.

We have also utilized six DCPs (digital potentiometers $\mathrm{X} 9 \mathrm{C} 102$, Xicor Inc.) in order to produce different resistivities (localized conductivity perturbations) in the phantom. The Ephantom has been divided into six segments where this variable resistivity can be applied.

We can change the resistivity of each segment of the planar electrode plate by using the variable resistor located between two nodes in each segment and imaging the conductivity perturbation in this segment.

\section{RSC MODEL}

The Cole-Cole impedance model is utilized to describe characteristics of the electrochemical properties of biological tissue with frequency dependence $[23,24]$. The model is created by using three electrical components of R, S (extra- and intra-cellular resistances) and $\mathrm{C}$ (membrane capacitance). In principle, it is sufficient to solve (1) for the sample tissue as well as the homogenous medium to obtain the frequency dependent model of the tissue. Fig. 3 shows the RSC circuit model that is incorporated into the E-phantom.

Thus including the frequency dependence of electrical parameters such as frequency dependence of conductivity $\left(\sigma_{\omega}\right)$, enables the model to capture the full range of physical phenomena.

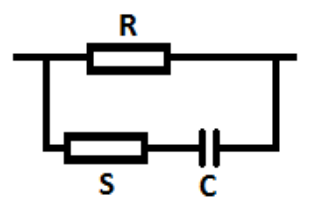

Fig. 3 Equivalent electrical circuit model for Cole-Cole equation
The impedivity data extracted from injection currents and voltage measurements in the EIT system when the RSC models are placed between electrodes in the E-phantom is compared with the impedance results that are determined using the ColeCole equation in order to assess the performance of the image reconstruction algorithm. This means simulating the impedance of different physical phenomena (in this case electrical properties of Carcinoma, Fat and Stroma tissues) at different frequency points $[14,25,26]$.

\section{Evaluation OF E-PHANTOM}

The Sussex EIT system consists of 85 electrodes and achieves 1416 independent measurement combinations based on 123 current injection channels. The image that is constructed using this large number of measurement combinations could mean this system should have the ability to make clearer images.[6, 27]. The Sussex EIT device achieves its measurement combinations for the planar electrode plate by using a hexagon structure based on a 4electrode method.

The constant current injector electrodes are located at the vertices of a hexagonal measurement shape. The hexagonal measurement structure is an experimental result that shows that a suitable drive pattern for the planar electrode plate is a hexagon combination with the 19 electrodes forming 3 injection channels using a 120 degree rotation around the $\mathrm{x}$ axis for each pair of electrode injections as shown in Fig. 4. The drive electrodes (e.g. in Fig. 4 the hexagon corners are e1-e19, e8-e12 and e3-e17 are drive electrodes) are always at a constant distance apart in any direction whereas the differential voltages are measured between any two electrodes. This process is continued for all electrodes. The image of 1416 voltage measurements of the conductivity (or impedivity) has been produced by image reconstruction software as shown in Fig. 4(b). This image is assumed to be ideal (back projection data) obtained from the dynamic measurement. This image was assigned to back projection data set. It is worth noting that the Sussex EIT system has a dynamic range of the voltage measurement frames as shown in Fig. 4(c). This plot simulated values of the 12 voltage measurements for a uniform conductivity medium. The model is for a homogenous distribution of saline with $0.5 \mathrm{mS} / \mathrm{m}$ conductivity and $1 \mathrm{mAp}-\mathrm{p}$ current injection. That's why the image obtained from the data of the phantom simulation representing a uniform case with transverse variation (see Fig. 4(b)) gives a method to help us to know the position of the error.

This mesh phantom is used to assess the performance of the EIT system to simulate in vivo conditions. The mesh phantom has the capability to generalize the different RSC models at 6 common locations with the capability to using a hexagonal topology in the planar structure at multiple frequencies. The RSC models are used to mimic the electrical properties of tissues inside a medium where the complex conductivity differs from the conductivity of the medium. 


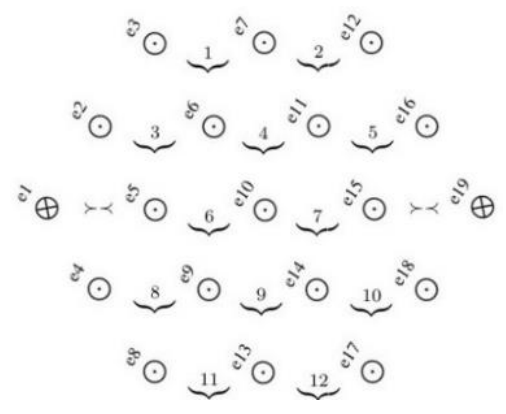

(a)

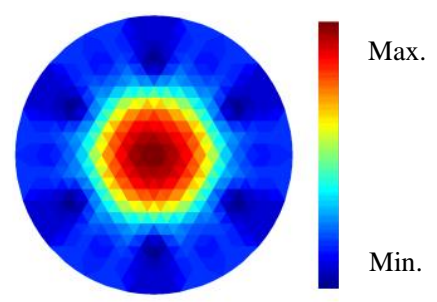

(b)

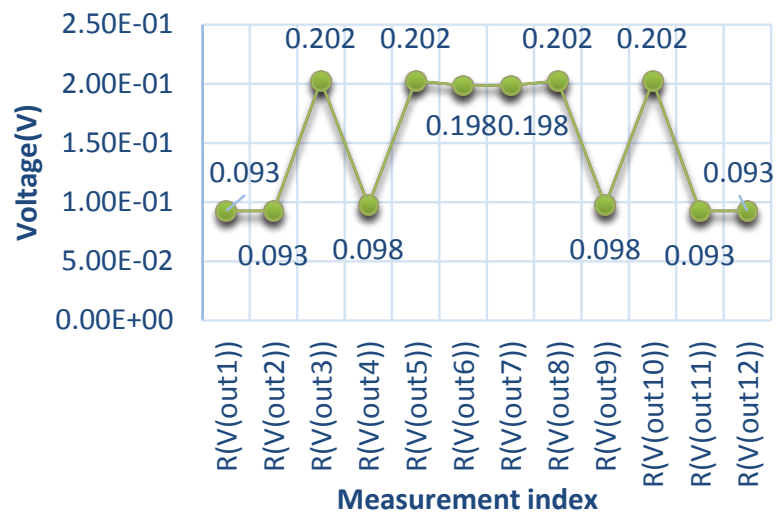

(c)

Fig. 4(a) A hexagon combination table consists of a 19 electrodes mesh. The current injection electrodes are denoted by [Q] and the voltage measurement electrodes are denoted by $[\odot]$, giving 12 measurement combinations for each injection channel, (b) an image that is made by the inversion created from the planar electrode plate connected to E-phantom as created from 1416 dynamic measurements of the homogenous saline medium, (c) the 12 voltage measurement indexes: $\mathrm{R}\left(\mathrm{V}_{\text {OUT1 }}\right)-\mathrm{R}\left(\mathrm{V}_{\text {OUT12 }}\right)$ when a current injection combination: e1-e19 of a hexagon combination table consists of 19 electrodes. Evidently the Sussex EIT has a good dynamic range of the voltage measurement frames.

As a next step, the theoretical result of the impedance measurements has been found by solving the Cole-Cole equation and matched with the experimental results that have been achieved.

Fig. 5 shows the impedance curve of three different tissues at different frequencies and impedance that has been achieved using the Cole-Cole equation. In general these curves are compared the theoretical and experimental results in the EIT system. Since, the objective of theory values is to find the actual behaviour of the system.

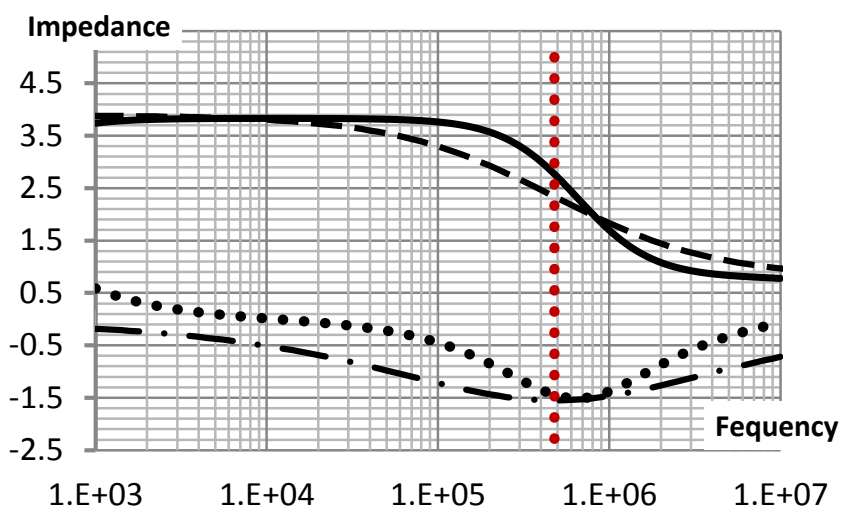

(a) Carcinoma

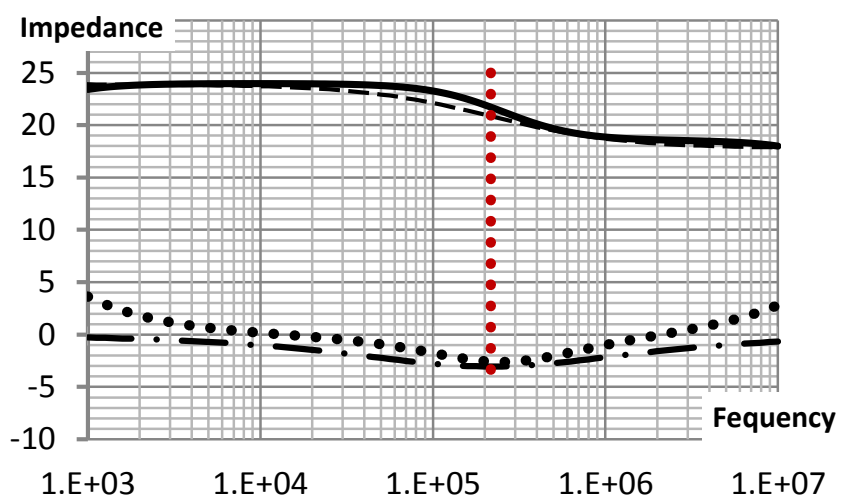

(b) Fat

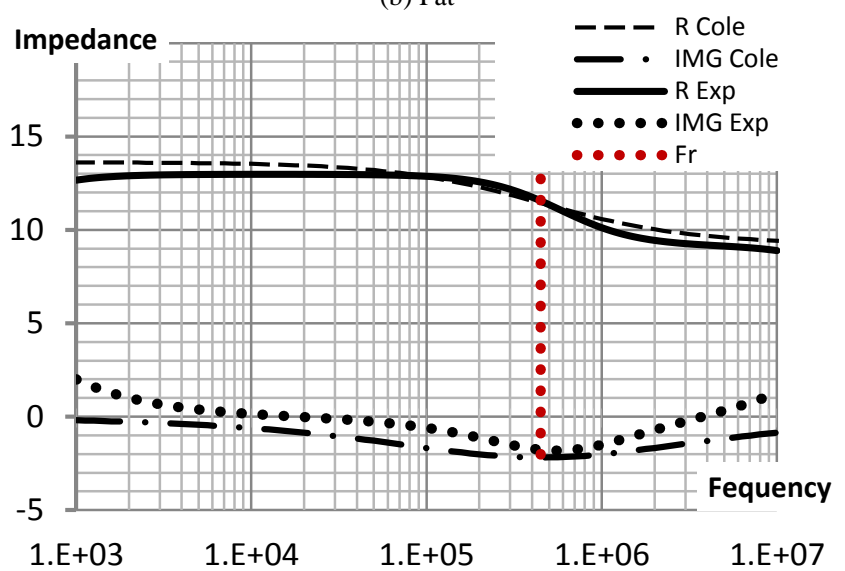

(c) Stroma

Fig. 5 Theoretical impedance spectra of Real and imaginary and relaxation frequency of (a) Carcinoma, (b) Fat and (c) Stroma tissues have been achieved by solving the Cole-Cole equation and experimental impedance curves have been achieved through connecting E-phantom to EIT board.

The mesh phantom is primarily employed to be connected instead of a physical phantom to assess the performance of an EIT system demonstrating the effect of a multi-frequency input signals. Fig.6 shows comparative images that have been produced by removing the back projection of the image. If we assumed the image without any RSC model is the back projection data, the removal of this data from the image during the reconstruction process will produce the final image that is shows the electrical properties of the RSC model between two electrodes. 


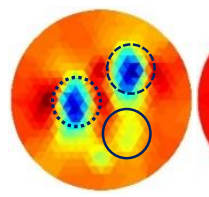

real,

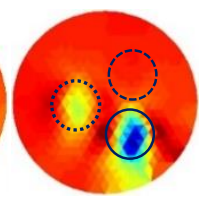

imaginary

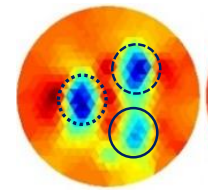

real,

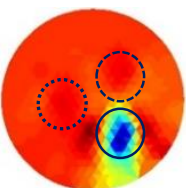

imaginary
Fig. 6 The real and imaginary part of frequency-difference images of an Ephantom within three RSC models RSC-1: placed at 17-5 (9 o'clock with dotted line) for carcinoma tissue, RSC-2: placed at 15-21 (2 o'clock with dashed line) for stroma tissue, and RSC-3: 19-30 (5 o'clock with lined) for fat tissue for $1 \mathrm{MHz}, 3 \mathrm{MHz}$ respectively.

The frequency bandwidth of the measurement sequence in this test is from $10 \mathrm{kHz}$ to $3 \mathrm{MHz}$.

In practice we inserted RSC models of the carcinoma, stroma and fat tissues in three different locations at 9, 2, and 5 O'clock to simulate the electrical properties of different tissues (with the RSC value based on Surowiec study [28]), then we constructed a tomographic image to reconstruct the real and imaginary parts for two sample frequency points of $1 \mathrm{MHz}$ and $3 \mathrm{MHz}$ in the Fig. 6.

The final step of the assessment of the EIT performance is measuring the SNR and modelling accuracy of the system. The Sussex EIT board with the 85 electrodes directly connected to E-phantom delivered an average SNR of $82.28 \mathrm{~dB}$ with a max and min value of $91.06 \mathrm{~dB}$ and $76.42 \mathrm{~dB}$. An average modelling accuracy of $99.47 \%$ with a max and min value of 99.97 and 99.91 was achieved.

\section{CONCLUSION}

The preliminary results have demonstrated that the Ephantom can be very effective as a clinical prototype for QA assessment and future certification. The purpose of the phantom is intended for system validation and performance testing during all phases of the clinical trials: pre-trial, during trial and future clinical derivatives. Moreover, utilizing DCPs in different segments of this E-phantom produces localized conductivity perturbations in order to evaluate the performance of image reconstruction algorithms.

\section{REFERENCES}

[1] D. S. Holder, Electrical Impedance Tomography: Methods, History and Applications. Bristol: Institute of Physics Publishing, 2005.

[2] B. Brown, "Electrical impedance tomography (EIT): a review," J. Med. Eng. Technol., vol. 27, pp. 97-108, 2003.

[3] Y. Zou and Z. Guo, "A review of electrical impedance techniques for breast cancer detection," Med. Eng. Phys., vol. 25, pp. 79-90, 2003.

[4] A. Zarafshani, N. Huber, N. Béqo, B. Tunstall, G. Sze, C. Chatwin and W. Wang, "A flexible low-cost, high-precision, single interface electrical impedance tomography system for breast cancer detection using FPGA," in Journal of Physics: Conference Series, 2010, pp. 012169.

[5] A. Zarafshani, T. Bach, W. Wang and C. Chatwin, "Conditioning a current source using OCCII-GIC for EIT systems," 2014

[6] G. Sze, "Detection of breast cancer with electrical impedance mammography, Ph.D. dissertation," 2012.

[7] Xiaolin Zhang, Wei Wang, G. Sze, D. Barber and C. Chatwin, "An Image Reconstruction Algorithm for 3-D Electrical Impedance Mammography," Medical Imaging, IEEE Transactions On, vol. 33, pp. 2223-2241, 2014.
[8] G Sze, W Wang, D C Barber and N Huber, "Preliminary study of the sensitivity of the sussex Mk4 electrical impedance mammography planar electrode system," in Bath, UK, 2011.

[9] T. K. Bera and J. Nagaraju, "A MATLAB-based boundary data simulator for studying the resistivity reconstruction using neighbouring current pattern," Journal of Medical Engineering, vol. 2013, 2013.

[10] H. Gagnon, A. E. Hartinger, A. Adler and R. Guardo, "A phantom for assessing the performance of EIT systems," in EIT Conf. 2008 (Dartmouth, NH, USA), 2008.

[11] G. Hahn, A. Just, J. Dittmar and G. Hellige, "Systematic errors of EIT systems determined by easily-scalable resistive phantoms," Physiol. Meas., vol. 29, pp. S163, 2008.

[12] H. Gagnon, M. Cousineau, A. Adler and A. E. Hartinger, "A resistive mesh phantom for assessing the performance of EIT systems," Biomedical Engineering, IEEE Transactions On, vol. 57, pp. 2257-2266, 2010.

[13] T. K. Bera and J. Nagaraju, "A chicken tissue phantom for studying an electrical impedance tomography (EIT) system suitable for clinical imaging," Sensing and Imaging: An International Journal, vol. 12, pp. 95-116, 2011.

[14] G. Qiao, W. Wang, L. Wang, Y. He, B. Bramer and M. Al-Akaidi, "Investigation of biological phantom for 2D and 3D breast EIT images," in 13th International Conference on Electrical Bioimpedance and the 8th Conference on Electrical Impedance Tomography, 2007, pp. 328-331.

[15] T. K. Bera, "Bioelectrical Impedance Methods for Noninvasive Health Monitoring: A Review," Journal of Medical Engineering, vol. 2014, 2014.

[16] T. Kao, G. J. Saulnier, D. Isaacson, T. L. Szabo and J. C. Newell, "A versatile high-permittivity phantom for EIT," Biomedical Engineering, IEEE Transactions On, vol. 55, pp. 2601-2607, 2008

[17] H. Griffiths, "A Cole phantom for EIT," Physiol. Meas., vol. 16, pp. A29, 1995.

[18] L. Fabrizi, A. McEwan, T. Oh, E. Woo and D. Holder, "A comparison of two EIT systems suitable for imaging impedance changes in epilepsy," Physiol. Meas., vol. 30, pp. S103, 2009.

[19] A. E. Hartinger, H. Gagnon and R. Guardo, "Accounting for hardware imperfections in EIT image reconstruction algorithms," Physiol. Meas., vol. 28, pp. S13, 2007.

[20] B. Rigaud, N. Chauveau, B. Ayeva, F. Fargues, E. Martinez and J. Morucci, "Modular cole phantom for parametric electrical impedance tomography," in Engineering in Medicine and Biology Society, 1996. Bridging Disciplines for Biomedicine. Proceedings of the 18th Annual International Conference of the IEEE, 1996, pp. 794-795.

[21] I. Schneider, R. Kleffel, D. Jennings and A. Courtenay, "Design of an electrical impedance tomography phantom using active elements," Medical and Biological Engineering and Computing, vol. 38, pp. 390394, 2000.

[22] H. Gagnon, Y. Sigmen, A. E. Hartinger and R. Guardo, "An active phantom to assess the robustness of EIT systems to electrode contact impedance variations," in Proceedings of the International Conference on Biomedical Applications of Electrical Impedance Tomography (EIT'09), 2009

[23] K. S. Cole and R. H. Cole, "Dispersion and absorption in dielectrics I Alternating current characteristics," J. Chem. Phys., vol. 9, pp. 341-351, 1941.

[24] K. S. Cole and R. H. Cole, "Dispersion and absorption in dielectrics II. Direct current characteristics," J. Chem. Phys., vol. 10, pp. 98-105, 1942.

[25] J. Jossinet, "The impedivity of freshly excised human breast tissue," Physiol. Meas., vol. 19, pp. 61, 1998.

[26] W. Wang, M. Tang, M. McCormick and X. Dong, "Preliminary results from an EIT breast imaging simulation system," Physiol. Meas., vol. 22 pp. 39, 2001.

[27] W. Wang, L. Wang, G. Qiao, P. Prickett, B. Bramer, B. Tunstall and M. Al-Akaidi, "Study into the repeatability of the electrode-skin interface utilizing electrodes commonly used in electrical impedance tomography," in 13th International Conference on Electrical Bioimpedance and the 8th Conference on Electrical Impedance Tomography, 2007b, pp. 336-339.

[28] A. J. Surowiec, S. S. Stuchly, J. R. Barr and A. Swarup, "Dielectric properties of breast carcinoma and the surrounding tissues," Biomedical Engineering, IEEE Transactions On, vol. 35, pp. 257-263, 1988. 